\title{
Coronavirus Disease-2019 and More: The Story of Coronaviruses So Far
}

\author{
Malavalli V Bhavana ${ }^{1}$, Apurva S Amarnath², Satish K Amarnath ${ }^{3}$
}

\begin{abstract}
Coronaviruses are not new to the human population. These enveloped ribonucleic acid (RNA) viruses have been the cause for various respiratory and gastrointestinal infections. They have caused serious epidemics of severe acute respiratory syndrome (SARS) and Middle East respiratory syndrome (MERS) earlier. Now, we are battling the current pandemic coronavirus disease-2019 (COVID-19). Though this novel virus has relatively low fatality, it has engulfed the word due to its high infectivity. This review is an attempt to take you through the story of coronaviruses so far, highlighting their virology, evolution, and spectrum of diseases. We have reviewed the effects of COVID-19 on pregnancy, newborns and children, testing and preventive strategies, herd immunity, and the way forward in this ongoing battle.
\end{abstract}

Keywords: Coronavirus, Coronavirus disease-2019, Severe acute respiratory syndrome coronavirus 2.

Pediatric Infectious Disease (2020): 10.5005/jp-journals-10081-1265

\section{INTRODUCTION}

A lot has been written and spoken about this pandemic which is ravaging the world. This virus has now engulfed the globe and has caused a huge economic burden for the world to face in the coming months. The world's medical professionals are in the forefront in this onslaught against the virus, yet the sheer numbers are swamping the medical facilities of even those countries with good medical services. This review takes you through the history, current taxonomy, the pathogenic spectrum, testing strategy of coronaviruses, with special reference to coronavirus disease-2019 (COVID-19). An insight about COVID-19 in pregnancy and in children is also provided.

The history of coronaviruses dates back to 1930s. They were implicated as causative agents of a respiratory infection of domesticated chickens. Human coronaviruses (HCoVs) were first described in the 1960s. They were known to cause upper respiratory infections, predominantly in children. The field of coronavirology has evolved over the years. The severe acute respiratory syndrome (SARS) epidemic in 2002 put these viruses in the spotlight. This was further accentuated by the Middle East respiratory syndrome (MERS) epidemic in 2012. The worst was yet to come. We are now battling a global health crisis. This is caused by a novel strain of coronavirus called the severe acute respiratory syndrome coronavirus 2 (SARS-CoV-2). Once an inconspicuous part of medical textbooks, now have reached pandemic proportions. Coronaviruses are no longer considered as innocuous respiratory viruses.

\section{Current Taxonomy}

According to the International Committee of Taxonomy of Viruses, coronaviruses belong to the order Nidovirales. This includes Coronaviridae, Arteriviridae, and Roniviridae families. All viruses belonging to this order are enveloped, non-segmented, and are positive-sense ribonucleic acid (RNA) viruses. Coronaviridae have the largest identified RNA genomes, comprising approximately $30 \mathrm{~kb}$. Coronaviridae family is further divided into four genera: Alphacoronavirus, Betacoronavirus, Gammacoronavirus, and Deltacoronavirus. ${ }^{1}$ Four distinct lineages (A, B, C, and D) have
'Department of Microbiology, Manipal Hospital, Bengaluru, Karnataka, India

${ }^{2}$ Department of Reproductive Medicine, NOVA IVF Fertility, Bengaluru, Karnataka, India

${ }^{3}$ Department of Quality, Compliance and Outreach Services, Manipal Hospital, Bengaluru, Karnataka, India

Corresponding Author: Malavalli V Bhavana, Department of Microbiology, Manipal Hospital, Bengaluru, Karnataka, India, Phone: +918025023355, e-mail: bhavana224@gmail.com

How to cite this article: Bhavana MV, Amarnath AS, Amarnath SK. Coronavirus Disease-2019 and More: The Story of Coronaviruses So Far. Pediatr Inf Dis 2020;2(2):55-61.

Source of support: Nil

Conflict of interest: None

been assigned within the genus Betacoronavirus. Among these, alphacoronaviruses and betacoronaviruses infect only mammals. The gammacoronaviruses and deltacoronaviruses usually infect birds, but some of them can also infect mammals. Alphacoronaviruses and betacoronaviruses mostly cause respiratory illnesses in humans and gastroenteritis in animals. ${ }^{2}$

There are six known HCoVs. Among them, HCoV-229E and HCoV-NL63 belong to the genus Alphacoronavirus, HCoV-OC43 and HCoV-HKU1 belong to lineage A, SARS coronavirus (SARS-CoV) to lineage $B$, and MERS-CoV to lineage $C$ of the genus Betacoronavirus. ${ }^{2}$

\section{Structure}

The virions are spherical, having a diameter of approximately $125 \mathrm{~nm} .^{3}$ The distinct feature is the presence of club-shaped spike projections from their surface. These spikes provide an appearance of the solar corona, due to which the name coronaviruses emerged. The nucleocapsid is present within the envelope. Coronaviruses have helically symmetrical nucleocapsids. ${ }^{4}$

\section{Evolution}

The history of HCoVs dates back to the year 1965 when Tyrrell and Bynoe found that they could passage a virus called B814. This 
virus was grown from human embryonic tracheal organ cultures from the respiratory tract of an adult with a common cold. The culture medium was inoculated intranasally in human volunteers. Colds were produced in a significant proportion of subjects after inoculation. Tyrrell and Bynoe were unable to grow the agent in tissue culture at that time. ${ }^{5}$

Two more scientists, Hamre and Procknow were able to grow a virus with unusual properties in tissue culture from samples obtained from medical students with colds at the same time period. They observed that both B814 and Hamre's virus, which they named as $229 \mathrm{E}$, were ether-sensitive. They inferred that a lipid-containing coat was required for infectivity. ${ }^{6}$

Later, Mclntosh, who was working in the laboratory of Robert Chanock at the National Institutes of Health, reported the recovery of multiple strains of ether-sensitive agents from the human respiratory tract. He and his team used a technique similar to that of Tyrrell and Bynoe. These viruses were termed "OC" to depict that they were grown in organ cultures. ${ }^{7}$

Almeida and Tyrrell performed electron microscopy on fluids from organ cultures at the same time. The organ cultures were infected with B814. They found particles that looked like the infectious bronchitis viruses of chickens. The particles were medium sized (80-150 nm), pleomorphic, membrane-coated, and covered with widely spaced club-shaped surface projections. They inferred that the 229E agent identified by Hamre and Procknow and the previous OC viruses identified by Mclntosh et al. were similar in morphology. ${ }^{8}$

The breakthrough came in the late 1960s. Tyrrell was leading a group of virologists doing research work on the human strains and animal viruses. These viruses included infectious bronchitis virus, mouse hepatitis virus, and transmissible gastroenteritis virus of swine. All of them had been demonstrated to be morphologically the same using electron microscopy. ${ }^{9}$ This new group of viruses were named coronaviruses (corona denoting the crown-like appearance of the surface projections). This was later officially accepted as a new genus of viruses. ${ }^{10}$

The widely studied human strains since the time of discovery were OC43 and 229E. The strain OC43 was initially adapted to growth in suckling mouse brain and subsequently to tissue culture. Strain 229E was grown in tissue culture directly from clinical samples. These two viruses demonstrate periodicity. They are responsible for large epidemics occurring at 2-3-year intervals. Reinfection is also common with them. Infection could occur at any age, but it was most common in children. Fifteen percent of adult colds too were attributed to these viruses. ${ }^{11,12}$

\section{Pathogenic Spectrum}

Epidemiological and volunteer inoculation studies have revealed that coronaviruses were associated with various respiratory illnesses. Their pathogenicity was considered to be low. They were predominantly associated with upper respiratory infections and occasional cases of pneumonia in infants and young adults. ${ }^{13,14}$ These viruses also caused exacerbations of asthma in children and chronic bronchitis in adults and the elderly. ${ }^{15-17}$

Further research also revealed that coronaviruses could infect multiple species, including rats, mice, chickens, turkeys, calves, dogs, cats, rabbits, and pigs. Pathogenesis of all the disease states caused was found to be complex. This genus was found to be capable of a wide variety of disease mechanisms in different species. Human and animal coronaviruses were then classified into three broad groups based on their antigenic and genetic makeup. Group I contained virus $229 \mathrm{E}$ and other viruses, group II contained virus OC43, and group III was made up of avian infectious bronchitis virus and a number of related avian viruses. ${ }^{18}$

A new, severe acute respiratory syndrome, called SARS, emerged in 2002-2003 in southern China. It soon spread throughout the world with quantifiable speed. This coronavirus strain was grown in tissue culture and the genome sequencing was performed. This strain differed from any of the known human or animal coronaviruses. It was placed in a new group, along with a virus that was subsequently cultured from Himalayan palm civets, from which it probably had emerged.

During the 2002-2003 outbreaks, SARS infection was reported in 29 countries in North America, South America, Europe, and Asia. Overall 8,098 infected individuals were identified; there were 774 SARS-related fatalities. ${ }^{19}$ It still baffles how the virus entered the human population and whether the Himalayan palm civets were actually the natural reservoir for the virus. Sequence analysis was also performed on the virus present in Himalayan palm civets. It was found that a 29-nucleotide sequence which it had, was not found in most human isolates causing the SARS infection. Whether the deletion of the 29-nucleotide sequence played a role in the trans-species jump leading to its virulence, is not clear. Seroepidemiological studies were conducted among food market workers in areas where the SARS epidemic began. The results showed that $40 \%$ of wild animal traders and $20 \%$ of individuals who slaughter animals were seropositive for SARS. Interestingly, none had a history of SARS-like symptoms. ${ }^{20}$ They were probably exposed to a SARS-like virus, through their occupation, leading to asymptomatic infection. SARS coronavirus uses angiotensin-converting enzyme 2 (ACE2) as a receptor and primarily infects ciliated bronchial epithelial cells and type II pneumocytes. The SARS epidemic gave the whole new facet to the research on coronaviruses.

These dynamicity of this evolution clearly proves that coronavirus is not a stable virus and can adapt to become more virulent, even lethal, to humans.

The world was shaken by another outbreak in Saudi Arabia in 2012 which resulted in many deaths and spread first to other countries in the Middle East and then worldwide. In June 2012, another highly virulent and novel coronavirus, MERS-CoV, was isolated from the sputum of a male patient who died from acute pneumonia and renal failure in Saudi Arabia. Nosocomial infections were later reported, and international travel led to the transmission of MERS-CoV to countries outside of the Middle East. In May 2015, an outbreak of MERS occurred in South Korea due to an individual returning from the Middle East. Bats were thought to be the possible natural reservoirs of both SARS and MERS-CoV. Dromedary camels could also be the possible intermediary hosts, before dissemination to humans.

Direct contact with the intermediary host might be the route of infection to humans. Camel workers in Saudi Arabia with high prevalence of MERS-CoV infection may contribute to the transmission of MERS. Consumption of milk, urine, or uncooked meat could be the other routes of exposure.

These were presumed to have caused the exposure to humans from the camels, principally in the Arabian Peninsula, human-tohuman spread was also detected later.

On similar lines, in December 2019, a new pneumonic disease began to appear in Wuhan, Hubei province, China. By early January 2020, the virus was identified and the sequence determined. The novel strain was named as SARS-CoV-2 and the disease caused was 
named as COVID-19. This strain shares $88 \%$ sequence homology to two coronaviruses found in bats, $79 \%$ identity with the SARS coronavirus, and $50 \%$ identity with MERS coronavirus. Coronavirus disease 2019 is characterized by a heterogeneous clinical course. While a majority of the patients experience only mild symptoms, a few develop severe disease, with increasing hypoxia progressing up to acute respiratory distress syndrome.

The function of the proteins in this virus has been extensively studied. The structural proteins of the virus are: spike protein (S), envelope protein (E), membrane protein $(M)$, and nucleocapsid phosphoprotein. Transcribed non-structural proteins include ORF1ab, ORF3a, ORF6, ORF7a, ORF10, and ORF8. ${ }^{21}$ There are studies which indicate that the damage of the virus to the human body is systemic. It is not confined only to the respiratory system. The structure of protein molecules such as ORF8 and surface glycoproteins were obtained using homology modeling methods and are being extensively studied. The study results showed that ORF8 and surface glycoproteins could combine to the porphyrin to form a complex. At the same time, ORF1ab, ORF10, and ORF3a proteins coordinate attack the heme on the 1-beta chain of hemoglobin to dissociate the iron to form the porphyrin. The attack decreases the efficiency of hemoglobin to carry oxygen and carbon dioxide. The lung cells get subjected to extremely intense inflammation due to the inability to exchange carbon dioxide and oxygen frequently, which eventually results in ground-glass-like lung images. The lung cells are further damaged by the free iron which is released into the bloodstream.

Diabetic patients and older people have higher glycated hemoglobin. Glycated hemoglobin was reduced by the attack, which makes the blood sugar unstable. This hypothesis warrants further extensive studies, but this is a valid explanation for the symptomatology seen in COVID-19 patients. $^{22}$

Severe COVID-19 is characterized by pneumonia, lymphopenia, exhausted lymphocytes, and a cytokine storm. Lymphopenia is a common occurrence, with drastically reduced numbers of $\mathrm{CD} 4^{+} \mathrm{T}$ cells, CD8 ${ }^{+} \mathrm{T}$ cells, B cells, and natural killer (NK) cells, as well as a reduced percentage of monocytes, eosinophils, and basophils. An increase in neutrophil count and in the neutrophil-to-lymphocyte ratio usually indicates disease severity. There are substantially elevated serum levels of proinflammatory cytokines including IL-6, IL-1 $\beta$, IL-2, IL-8, IL-17, G-CSF, GM-CSF, IP10, MCP1, MIP1a (also known as (CL3), and TNF, causing a cytokine storm. The resulting lung inflammation and fluid build-up can lead to respiratory distress and a secondary bacterial pneumonia. This eventually leads to shock and multiorgan failure. ${ }^{23-25}$

\section{Coronavirus Disease-2019 and Pregnancy}

It is a known fact that respiratory viral infections predispose a woman to adverse obstetric and neonatal outcomes. There is increased risk of respiratory disease and its complications in view of the physiological and immunological adaptations that occur in all normal pregnancies. ${ }^{26}$ A normal pregnancy has changes that occur in both the cardiovascular and respiratory systems like changes in heart rate, stroke volume, increased oxygen consumption, and also decrease in the total lung capacity along with immunological changes that occur in women which allow for the antigenic acceptance of the fetus which is distinct from herself-all of these contribute to a pregnant women being at higher risk of developing severe disease. ${ }^{20}$ Additionally, association with comorbidities, such as gestational diabetes and other immunocompromised states, may contribute to increase disease severity. Early studies with small sample sizes have shown that there is no difference in the clinical presentation and outcomes of pregnant women infected with COVID-19 vs that of the general population, but due to sample sizes concrete conclusions cannot be drawn. ${ }^{19}$

As the pandemic rages on, there is likelihood of increased numbers of pregnant women being affected with SARS-CoV-2, hence maternal management and fetal safety is of concern.

It is essential to know if pregnant women are more susceptible to the virus, and are the symptoms of pneumonia in pregnant women different from those of nonpregnant women.

\section{Maternal Outcomes}

\section{First Trimester}

As we are still in the early stages of disease, much is not known about the effects of COVID-19 infection in the first trimester. Data on whether there is any effect on pregnancy in the stage of organogenesis, the rate of vertical transmission, and the risk of teratogenicity of the virus on the growing fetus need to be assessed.

\section{Second/third Trimester}

There are scant data on second trimester of pregnancy currently. Most data come from the third trimester of pregnancy. In a study comprising 32 women, 2 women developed serious morbidity with COVID-19 requiring ICU care. ${ }^{27}$ Coronavirus disease 2019 appears less severe at this point in comparison to SARS and MERS, but further data would be required to make conclusive inferences.

\section{Fetal and Placental Effects}

Most studies regarding COVID-19 in pregnancy are from the late third trimester and have a short time from infection-delivery duration with a mean duration of 13 days in which time duration, fetal growth is not going to be affected..$^{19,28,29}$ There is no data on the placental pathology. Further data are needed to understand more.

\section{Delivery and Postnatal Period}

In a study by Zhu et al., reporting on 9 women who delivered 10 neonates, it was noted that 7 women delivered by cesarean section and 2 vaginally and only 3 of the mothers were symptomatic after delivery. These women had COVID-19 from the gestational age of 31 weeks onward, $55 \%$ of the pregnancies showed fetal distress and $55.5 \%$ of women ( $60 \%$ babies) delivered preterm - which is of concern due to increased morbidity that is noted in preterm babies. $^{30}$

As per a study by Mullins et al., "From reports of 32 women to date affected by COVID-19 in pregnancy, delivering 30 babies (one set of twins, three ongoing pregnancies), seven (22\%) were asymptomatic and two (6\%) were admitted to the intensive care unit (ICU), one of whom remained on extracorporeal membrane oxygenation. No maternal deaths have been reported to date. Delivery was by cesarean section in 27 cases and by vaginal delivery in two, and 15 (47\%) delivered preterm. There was one stillbirth and one neonatal death. In 25 babies, no cases of vertical transmission were reported; 15 were reported as being tested with reverse transcription polymerase chain reaction after delivery". ${ }^{27}$

\section{Neonatal Outcomes}

Few studies have been conducted to assess neonatal outcomes after delivery. In the study of Chen et al., the neonates delivered 
at $\geq 36$ weeks' gestation had no neonatal complications. ${ }^{19}$ As per Zhu et al., 55\% of deliveries occurred preterm (from 31 weeks); $6 / 10(60 \%)$ babies required neonatal intensive care unit (NICU), for respiratory support, with 2 babies developing disseminated intravascular coagulation (DIC) and 1 developing multiple organ failure. ${ }^{30}$ There was higher neonatal morbidity noted in this series, most likely due to higher prematurity. There was one mortality noted at 8 days postpartum after being born at 34 weeks. 9/10 (90\%) of the infants were tested for COVID-19, with no cases being reports among the infants. Wang et al. reported a baby born to a COVID-19 infected mother at 30 weeks with an uneventful neonatal course and was discharged healthy. ${ }^{28}$ As per Liu et al., it was noted that there was one stillbirth and nine live births, with no neonatal adverse events. ${ }^{29}$ As per the data available, neonatal morbidity is mainly increased due to prematurity, which could be spontaneous or iatrogenic, with no direct link to COVID-19 currently found. However, data regarding the fetal morbidity in women affected remote to term/delivery is still to be assessed.

The acquisition of infection in newborns is most likely due to close contact with infected mothers. Suspicion of COVID-19 in newborns should occur when they present with at least 1 clinical symptom which could be fluctuating body temperature, poor feeding or failure to thrive, respiratory symptoms, such as breathlessness. Other features include chest X-ray showing unilateral or bilateral milled glass opacities, and epidemiological features, such as COVID-19 infection diagnosis in the patient's family or caregivers or having close contact with people have confirmed COVID-19 infection or with patients with unexplained pneumonia. ${ }^{31}$

\section{Vertical Transmission}

There are studies which reported that there was no vertical transmission of infection. ${ }^{32}$ However, as per a recent report by Dong et al., it has been noted that a neonate born to a 28 -year-old primigravida who was a confirmed case of COVID-19, was tested for COVID-19 IgG and IgM antibodies at 2 hours of age. The IgM level was $45.83 \mathrm{AU} / \mathrm{mL}$ and the IgG level was $140.32 \mathrm{AU} / \mathrm{mL}$. The nasopharyngeal swabs from the neonate for real time polymerase chain reaction (RT-PCR) were negative. Since IgM does not cross the placenta and the testing was performed early after delivery and the fact that IgM takes 3 to 7 days to appear in the bloodstream after infection, the presence of IgM suggests that there was in utero transmission of infection. ${ }^{33}$

It is to be noted that there is paucity of data in view of vertical transmission; widespread studies are needed for the same to evaluate the vertical transmission rates and to assess the effect of the same depending on the period of gestation especially in view of teratogenicity.

To summarize, the possibility of pregnancy as a risk factor for increased disease severity with COVID-19 cannot be conclusively postulated in view of the scant data. It is know that comorbidities, such as diabetes, cardiac failure, or hypertension, risk factors for severe COVID-19 infections in adults (non-pregnant), and it needs to be evaluated if these would be additional risk factors for pregnant women. ${ }^{34}$ The outcomes of infants born to COVID-19 mothers also seem to be fairly good. However, it has to be noted that the data set studied is small in most of the studies and deliveries occurred at late stages where the fetal outcome is expected to be good. Experience with previous SARS virus has shown that there is increased risk of adverse outcomes, such as intrauterine growth restriction, preterm birth, intrauterine death, and neonatal death. ${ }^{35}$
Therefore, rigorous screening of pregnant women and suspected women needs to be performed and follow-up for pregnancy outcome and neonatal outcome needs to be maintained. The risk of vertical transmission also needs to be investigated more so as they have a complete clinical picture to aid in counseling the prospective parents.

\section{Coronavirus and Children}

Seasonal coronaviruses contribute to $4-6 \%$ of acute respiratory tract infections needing hospitalization in children. It commonly occurs in children $<3$ years of age and in children with congenital heart disease. Routine reinfection later in life is also commonly seen with coronaviruses. Other respiratory tract viruses like respiratory syncytial virus (RSV) show a decrease in prevalence with age, which is not seen with the coronavirus, with the relative prevalence staying the same. In children, common circulating coronaviruses can cause fever, rhinitis, otitis, pharyngitis, laryngitis, bronchitis, bronchiolitis, and headache. ${ }^{36}$

\section{Coronavirus Disease-2019 and Children}

Several studies have been conducted to understand the impact of COVID-19 in children. There is need to study the manifestations and effects of the virus in children as there are multiple conditions causing similar symptoms.

As per the study by Dong et al., data were procured from the Chinese Center for Disease Control and Prevention. A case series of 2,143 pediatric patients, nationwide were included. The cases were diagnosed by clinical and laboratory criteria. ${ }^{37}$ "One epidemiological criteria needed to be met for suspected COVID19 (i.e., children with travel history or contact with patients with fever or respiratory symptoms with travel history to areas with persistent local transmission and newborns delivered by confirmed 2019-nCoV-infected mothers) and any two of the clinical criteria (fever, fatigue, dry cough with chest imaging findings like multiple small plaques and interstitial changes, bilateral multiple ground glass opacity and/or infiltrating shadows, lung consolidation, pleural effusion)". ${ }^{38}$

"Suspected cases who test positive for 2019-nCoV nucleic acid using RT-PCR or a positive genetic sequencing of respiratory tract or blood samples is highly homologous with the known 2019-nCoV are considered cases". ${ }^{37}$

The affected children can clinically fall into any of the following groups: ${ }^{39}$

- Asymptomatic infection (silent infection): Children tested positive for the virus, with absence of clinical symptoms or abnormal chest imaging findings.

- Mild disease: Symptoms of acute respiratory tract infection are seen like fever, cough, pharyngeal pain, nasal congestion, fatigue, headache, myalgia or discomfort, etc., in the absence of signs of pneumonia by chest imaging or sepsis.

- Moderate disease: Pneumonia is seen with or without fever, respiratory symptoms, such as cough; but no hypoxemia or altered sensorium noted.

- Severe disease: This is characterized by:

- Increased respiratory rate: $\geq 70$ times/min ( $<1$ year), $\geq 50$ times/ $\min$ ( $\geq 1$ year) (after ruling out the effects of fever and crying).

- Oxygen saturation $<92 \%$. 
- Hypoxia: Assisted breathing (moans, nasal faring, and three concave sign), cyanosis, intermittent apnea.

- Disturbance of consciousness: Somnolence, coma, or convulsion.

- Food refusal or feeding difficulty, with signs of dehydration.

- Critical cases: Those who meet any of the following criteria and require ICU care:

- Acute respiratory distress syndrome requiring mechanical ventilation.

- Shock.

- Multiorgan failure. ${ }^{39}$

A study on COVID-19 in children examined 134 patients whose clinical data were available among 285 pediatric cases (as of February 7, 2020) diagnosed in China. Fever and cough were the most common symptoms; other clinical features included fatigue, myalgia, rhinorrhea, nasal congestion, sore throat, headache, dizziness, nausea, vomiting, abdominal pain, and diarrhea. Symptom resolution was usually seen in a week. Out of the 134 children, temperature was recorded for 117 of them, 89 children had a fever that lasted usually 1-2 days but up to 8 days. The complete blood counts were within the normal range in most patients with slightly decreased lymphocyte count. C-reactive protein (CRP) was normal or temporarily increased slightly. Of the 134 cases, 9 patients were asymptomatic with normal chest radiology findings, 87 had mild symptoms with normal chest radiology findings, 36 had pneumonia, and 2 were critically ill (both of whom were mechanically ventilated). Both the critical patients had associated comorbidities. Fifty-four patients had chest radiology data available of which ground-glass opacity, exudative/infiltrative lesions, increased pulmonary markings were mostly observed. ${ }^{40}$ In children with severe cases, association with septic shock, metabolic acidosis, and DIC can occur. ${ }^{41,42}$

The initial presenting clinical and radiological features of COVID-19 in pediatric and adult patients undergoing CT scans for respiratory symptoms. There was less extensive lung involvement with number of pulmonary lobes involved in the pediatric age group being lower when compared with the adult population. There is higher incidence of peribronchial distribution and bronchial wall thickening, in the pediatric population when compared to the adult population. The radiological changes seen in the pediatric population are in the periphery of the lungs, close to the pleural surface-which aligns with the bronchovascular bundles, mimicking changes of bronchopneumonia. Though ground-glass opacities and consolidation also occur, the most characteristic changes seen in children are single lung involvement characterized by few nodular or small/patchy changes. Adult patients have more widespread changes in view of the robust inflammatory response in adults. Adult manifestations, such as nodal enlargement and pleural effusions, have not been seen in children. The lesions in the pediatric population usually resolves after treatment, without long-term sequelae, and currently with no evidence of relapse; whereas, in adults, fibrous cords and secondary diseases may develop. ${ }^{36,43}$

Children form a small demographic sector of the affected, with a large proportion being symptomatic or having mild-moderate symptoms. Fatality also is low in children, with severity being highest in infants. However, some pediatric cases may progress to severe disease, and initial atypical presentations may delay the diagnosis of COVID-19, leading to unfavorable outcomes. Also, as there are many conditions in children which mimic the symptoms of COVID-19, there is danger of both under diagnosis and over diagnosis. Further studies to throw more light on the reason for differences in clinical features of COVID-19 by age and the role of children in community transmission are the need of the hour.

\section{Testing Strategy for Coronavirus DISEASE-2019}

The approach toward testing depends on patient selection, test selection to confirm or rule out the infection and for patient monitoring.

The selection of appropriate patients for testing is subject to statutory guidelines. It is advisable to go through the updated guidelines on the health ministry website to select the appropriate patient for testing. ${ }^{44} \mathrm{~A}$ thorough history and clinical assessment is crucial.

The laboratory tests could be specific and nonspecific. The nonspecific blood markers include lymphopenia, mild thrombocytopenia, raised lactate dehydrogenase (LDH), creatine kinase (CK), CRP, and erythrocyte sedimentation rate (ESR). ${ }^{45}$

It is, however, noteworthy to mention in children, that the white blood cell (WBC) count could be normal or reduced with decreased neutrophil and/or lymphocyte counts. C-reactive protein and procalcitonin levels are often normal. In severe cases, elevated liver enzymes can be seen. ${ }^{36}$

Elevated ferritin, D-dimer, proinflammatory cytokines, urea, and creatinine are the indicators of severe infection. A raised neutrophil lymphocyte ratio (NLR) $\geq 3.13$ in patients $\geq 50$ years is another prognostic marker for disease severity. ${ }^{46}$ Increased IL-6 levels above $80 \mathrm{pg} / \mathrm{mL}$ suggest high risk of respiratory failure. ${ }^{47}$

As for the specific tests, the viral RNA detection by reverse transcriptase real time PCR (rRT-PCR) is currently advocated. Nasal and throat swab samples are to be collected in one viral transport media (VTM) tube. Both nares should be swabbed with a polyester or dacron swab with a plastic shaft. Oropharyngeal specimen, nasal mid-turbinate swab, and anterior nares (nasal swab) are the other alternatives. Among the lower respiratory samples, a deep coughed out sputum, endotracheal aspirate, and bronchoalveolar lavage are recommended. Induction of sputum and transporting the samples through the pneumatic tube system are not recommended. ${ }^{44,48}$

The samples collected are shipped after a triple-layered packing at $2-8^{\circ} \mathrm{C}$.

First-line screening assay targets the SARS-CoV-2-specific E gene. Confirmatory assay usually targets the RdRp gene, $\mathrm{N}$ gene, and ORF-1b. The probes are primers are designed for the specific target gene. ${ }^{49}$

In addition, the cartridge-based nucleic acid amplification technique is now approved for the RNA detection (CBNAAT). This has a shorter turnaround time compared to the polymerase chain reaction $(\mathrm{PCR}){ }^{50}$

It is to be remembered that the sensitivity of PCR is $67 \%$ between days 1 and 7 of symptom onset. This further decreases with the passage of time. ${ }^{51}$

The IgM and IgG antibody tests which measure the immune response have also come into forefront. The usage has been restricted to serosurveillance among healthcare workers. Their utility may widen in the near future. ${ }^{44}$ Immunochromatography, enzymelinked immunosorbent assay (ELISA), and chemiluminescence are the different platforms on which this testing can be performed. The sensitivity of total antibody detection (IgM and IgG) in the second week of illness is $90 \%$, which further increases by third week. ${ }^{51}$ 
Yet, another technology in vogue in the United States is the reverse transcription loop-mediated isothermal amplification (RTLAMP). This uses isothermal nucleic acid amplification technology, which does not require a series of alternating temperature cycles. The results are very rapid. It is an FDA approved test. ${ }^{52}$

\section{Herd Immunity and the Way Forward}

Coronavirus disease 2019 is here to stay, at least until safe and effective vaccines become available. Immunization must be rolled out across the globe to limit the infection. As on today, one vaccine candidate is in a phase 2 trial, four in phase 1 trials, and 56 in preclinical studies. ${ }^{50}$

Even with advanced platforms, SARS-CoV-2 vaccine development poses challenges. Though virus's spike protein is a promising immunogen for protection, optimized antigen design is critical to ensure desired immune response. So, targeting the fulllength protein or only the receptor-binding domain is the debate that is ongoing. Also, testing in a suitable animal model and rigorous safety monitoring in clinical trials will be critical. ${ }^{53}$

It is worthwhile to debate whether low-risk age groups living in low-risk households could be allowed to return to the economy while maintaining a degree of social distancing to protect the vulnerable. Herd immunity through naturally acquired infection might increase until vaccination programs can start. It is still unknown till what duration does the immunity last after an infection. Reinfection is also a possibility as per current data. So, it is quite unclear on how to mitigate the way forward.

Before initiating any exit strategy, studies say that we need to estimate the basic reproductive number (R0), or the more "real-life" effective reproductive number (Rt) for a given population. R0 is the number of secondary cases generated by the presence of one infected patient in an otherwise healthy but fully susceptible, wellmixed population. Rt uses real-life data (from diagnostic testing and/or clinical/digital surveillance) to estimate the reproductive number for an ongoing epidemic. It is recommended to look into the data on the daily number of new COVID-19 cases, together with a recent estimate of the serial interval at a 0.05 significance level, with the mathematical software R (v3.6.1.) Using these values of Rt, it is recommended to calculate the minimum level of population immunity, Pcrit, acquired via vaccination or naturally induced (i.e., after recovery from COVID-19), to halt the spread of infection in that population, using the formula: Pcrit $=1-(1 / R t)$. So, for example, if the value of $\mathrm{Rt}=3$ then Pcrit $=0.67$. This means $67 \%$ of the total population should recover from the infection to confer immunity. There is also a fatality rate of $2-3 \%$ to account for in this process. ${ }^{54}$

\section{CONCLUSION}

The COVID-19 pandemic has devastated the world economy and has overwhelmed the health facilities in developed countries. It has certainly paralyzed the medical care delivery in most of the regions, leading to innovations like video consultations with interfaced instruments, redesigning of care delivery and new vistas for research and care delivery. There has been a swath of postponement of planned surgical and medical care; misunderstanding of the viral transmission leading to errors in judgment of care delivery planning. Every country is busy contemplating on a suitable exit strategy after prolonged lockdowns. As of now, vaccination seems to be the only clear solution we have. The rest of the strategies are subject to extensive debates and discussions. This pandemic has taught us that containment measures and aggressive contract tracing are mandatory to keep the situation under control. It is appreciated if a global pandemic control plan is formulated and initiated with appropriate financial scaling to tackle future such incidents. We are seeing a new era; this experience will reengineer the way we deliver care to a swath of the world's population. There is going to be huge learning as we conquer this pandemic.

\section{References}

1. Woo PCY, Lau SKP, Lam CSF, et al. Discovery of seven novel mammalian and avian coronaviruses in Deltacoronavirus supports bat coronaviruses as the gene source of Alphacoronavirus and Betacoronavirus and avian coronaviruses as the gene source of Gammacoronavirus and Deltacoronavirus. J Virol 2012;86(7):39954008. DOI: 10.1128/JVI.06540-11.

2. Fung TS, Liu DX. Human coronavirus: host-pathogen interaction. Annu Rev Microbiol 2019;73(1):529-557. DOI: 10.1146/annurevmicro-020518-115759.

3. Neuman BW, Adair BD, Yoshioka C, et al. Supramolecular architecture of severe acute respiratory syndrome coronavirus revealed by electron cryomicroscopy. J Virol 2006;80(16):7918-7928. DOI: 10.1128/ JVI.00645-06.

4. Fehr AR, Perlman S. Coronaviruses: an overview of their replication and pathogenesis. Methods Mol Biol 2015;1282:1-23. DOI: 10.1007/978-1-4939-2438-7_1.

5. Tyrrell DA, Bynoe ML. Cultivation of viruses from a high proportion of patients with colds. Lancet 1966;1(7428):76-77. DOI: 10.1016/s01406736(66)92364-6.

6. Hamre D, Procknow JJ. A new virus isolated from the human respiratory tract. Proc Soc Exp Biol Med 1966;121(1):190-193. DOI: 10.3181/00379727-121-30734.

7. Mclntosh K, Becker WB, Chanock RM. Growth in suckling-mouse brain of "IBV-like" viruses from patients with upper respiratory tract disease. ProcNatl Acad Sci USA 1967;58(6):2268-2273. DOI: 10.1073/ pnas.58.6.2268.

8. Almeida JD, Tyrrell DA. The morphology of three previously uncharacterized human respiratory viruses that grow in organ culture. J Gen Virol 1967;1(2):175-178. DOI: 10.1099/0022-13171-2-175.

9. Witte KH, Tajima M, Easterday BC. Morphologic characteristics and nucleic acid type of transmissible gastroenteritis virus of pigs. Arch Gesamte Virusforsch 1968;23(1-2):53-70. DOI: 10.1007/BF01242114.

10. Tyrrell DAJ, Almeida JD, Cunningham $\mathrm{CH}$, et al. Coronaviridae. Intervirology 1975;5(1-2):76-82. DOI: 10.1159/000149883.

11. Monto AS. Medical reviews: coronaviruses. Yale J Biol Med 1974;47(4):234-251.

12. Callow KA, Parry HF, Sergeant $M$, et al. The time course of the immune response to experimental coronavirus infection of man. Epidemiol Infect 1990;105(2):435-446. DOI: 10.1017/s0950268800048019.

13. Mclntosh K, Chao RK, Krause HE, et al. Coronavirus infection in acute lower respiratory tract disease of infants_see comment. J Infect Dis 1974;130(5):502-507. DOI: 10.1093/infdis/130.5.502.

14. Wenzel RP, Hendley JO, Davies JA, et al. Coronavirus infections in military recruits. Three-year study with coronavirus strains OC43 and 229E. Am Rev Respir Dis 1974;109(6):621-624. DOI: 10.1164/ arrd.1974.109.6.621.

15. McIntosh K, Ellis EF, Hoffman LS, et al. Association of viral and bacterial respiratory infection with exacerbations of wheezing in young asthmatic children. Chest 1973;63(suppl):43S. DOI: 10.1378/ chest.63.4_supplement.43s.

16. Falsey AR, McCann RM, Hall WJ, et al. The "common cold" in frail older persons: impact of rhinovirus and coronavirus in a senior daycare center. J Am Geriatr Soc 1997;45(6):706-711. DOI: 10.1111/j.15325415.1997.tb01474.x.

17. Falsey AR, Walsh EE, Hayden FG. Rhinovirus and coronavirus infection-associated hospitalizations among older adults. J Infect Dis 2002;185(9):1338-1341. DOI: 10.1086/339881. 
18. Haring J, Pearlman S. Mouse hepatitis virus. Curr Opin Microbiol 2001;4(4):462-466. DOI: 10.1016/s1369-5274(00)00236-8.

19. Chen $\mathrm{H}$, Guo J, Wang $\mathrm{C}$, et al. Clinical characteristics and intrauterine vertical transmission potential of COVID-19 infection in nine pregnant women: a retrospective review of medical records. Lancet 2020;395(10226):809-815. DOI: 10.1016/S0140-6736(20)30360-3.

20. Schwartz DA. An analysis of 38 pregnant women with COVID-19, their newborn infants, and maternal-fetal transmission of SARS-CoV-2: Maternal coronavirus infections and pregnancy outcomes. Arch Pathol Lab Med 2020(7). DOI: 10.5858/arpa.2020-0901-SAOnline ahead of print.

21. Wu F, Zhao S, Yu B, et al. A novel coronavirus associated with human respiratory disease in China. Nature 2020;579(7798):265-269. DOI: 10.1038/s41586-020-2008-3.

22. Wenzhong LIU, Hualan LI. COVID-19 disease: ORF8 and surface glycoprotein inhibit heme metabolism by binding to porphyrin. 2020. https://chemrxiv.org/ndownloader/files/22283226 accessed on 05.05.2020.

23. Huang C, Wang Y, Li X, et al. Clinical features of patients infected with 2019 novel coronavirus in Wuhan, China. Lancet 2020;395(10223): 497-506. DOI: 10.1016/S0140-6736(20)30183-5.

24. Xu Z, Shi L, Wang Y, et al. Pathological findings of COVID-19 associated with acute respiratory distress syndrome. Lancet Respir Med 2020;8(4):420-422. DOI: 10.1016/S2213-2600(20)30076-X.

25. Cao X. COVID-19: immunopathology and its implications for therapy. Nat Rev Immunol 2020;20(5):269-270. DOI: 10.1038/s41577-0200308-3.

26. Rasmussen SA, Jamieson DJ, Uyeki TM. Effects of influenza on pregnant women and infants. Am J Obstet Gynecol 2012;207(3 Suppl):S3-S8. DOI: 10.1016/j.ajog.2012.06.068.

27. Mullins $E$, Evans $D$, Viner RM, et al. Coronavirus in pregnancy and delivery: rapid review. Ultrasound Obstet Gynecol 2020;55(5):586592. DOI: 10.1002/uog.22014.

28. Wang X, Zhou Z, Jianping Z, et al. A case of 2019 novel coronavirus in a pregnant woman with preterm delivery. Clin Infect Dis 2020(15):ciaa200. DOI: 10.1111/aos.14584.

29. Liu Y, Chen H, Tang K, et al. Clinical manifestations and outcome of SARS-CoV-2 infection during pregnancy. J Infect 2020. pii: S01634453(20)30109-2.

30. Zhu H, Wang L, Fang C, et al. Clinical analysis of 10 neonates born to mothers with 2019-nCoV pneumonia. TransI Pediatr 2020;9(1):51-60. DOI: $10.21037 /$ tp.2020.02.06.

31. Hong $\mathrm{H}$, Wang $\mathrm{Y}$, Chung HT, et al. Clinical characteristics of novel coronavirus disease 2019 (COVID-19) in newborns, infants and children. Pediatr Neonatol 2020;61(2):131-132. DOI: 10.1016/j. pedneo.2020.03.001.

32. Karimi-Zarchi $M$, Zanbagh $L$, Javaheri $A$, et al. Vertical transmission of coronavirus disease 19 (COVID-19) from infected pregnant mothers to neonates: a review. Fetal Pediatr Pathol 2020. 1-5. DOI: 10.1080/15513815.2020.1745970.

33. Dong L, Tian J, He S, et al. Possible vertical transmission of SARS-CoV-2 from an infected mother to her newborn. JAMA 2020;323(18):18461848. DOI: $10.1001 /$ jama.2020.4621.

34. Li B, Yang J, Zhao F, et al. Prevalence and impact of cardiovascular metabolic diseases on COVID-19 in China. Clin Res Cardiol 2020;109(5):531-538. DOI: 10.1007/s00392-020-01626-9.

35. Lam CM, Wong SF, Leung TN, et al. A case-controlled study comparing clinical course and outcomes of pregnant and non-pregnant women with severe acute respiratory syndrome. BJOG 2004;111(8):771-774. DOI: 10.1111/j.1471-0528.2004.00199.x.

36. Zimmermann $\mathrm{P}$, Curtis N. Coronavirus infections in children including COVID-19: an overview of the epidemiology, clinical features, diagnosis, treatment and prevention options in children. Pediatr Infect Dis J 2020;39(5):355-368. DOI: 10.1097/INF.0000000000002660.

37. Dong Y, Mo X, Hu Y, et al. Epidemiological characteristics of 2143 pediatric patients with 2019 coronavirus disease in china. Pediatrics 2020;145(6):e20200702. DOI: 10.1542/peds.2020-0702.

38. Shen K, Yang Y, Wang T, et al. Diagnosis, treatment, and prevention of 2019 novel coronavirus infection in children: experts' consensus statement. World J Pediatr 2020;16(3):223-231. DOI: 10.1007/s12519020-00343-7.

39. National Health Commission of People's Republic of China. Diagnosis and treatment of pneumonia caused by novel coronavirus (trial version 4). https://www.nhc.gov.cn/xcs/zhengcwj/20200 accessed on 1st May 2020.

40. Society of Pediatrics, Chinese Medical Association; Editorial Board, Chinese Journal of Pediatrics. Recommendations for the diagnosis, prevention and control of the 2019 novel coronavirus infection in children (first interim edition). Zhonghua Er Ke Za Zhi 2020;58: 169-174.

41. Choi S-H, Kim HW, Kang J-M, et al. Epidemiology and clinical features of coronavirus disease 2019 in children. Clin Exp Pediatr 2020;63(4):125-132. DOI: 10.3345/cep.2020.00535.

42. Chen ZM, FuJF, Shu Q, etal.Diagnosis and treatment recommendations for pediatric respiratory infection caused by the 2019 novel coronavirus. World J Pediatr 2020(3):240-246. DOI: 10.1007/s12519020-00345-5.

43. Xia W, Shao J, Guo Y, et al. Clinical and CT features in pediatric patients with COVID-19 infection: different points from adults. Pediatr Pulmonol 2020;55(5):1169-1174. DOI: 10.1002/ppul.24718.

44. https://www.icmr.nic.in/content/covid-19.

45. Fan BE, Chong VCL, Chan SSW, et al. Hematologic parameters in patients with COVID-19 infection. Am J Hematol 2020;95(6):E131E134. DOI: 10.1002/ajh.25774.

46. Liu Y, Du X, Chen J, et al. Neutrophil-to-lymphocyte ratio as an independent risk factor for mortality in hospitalized patients with COVID-19. J Infect 2020;81(1):e6-e12. DOI: 10.1016/j.jinf.2020.04.002.

47. Herold T, Jurinovic V, Arnreich C, et al. Elevated levels of IL- 6 and CRP predict the need for mechanical ventilation in COVID-19. J AllergyClin Immunol 2020;146(1):128-136.

48. https://www.cdc.gov/coronavirus/2019-ncov/lab/guidelines-clinicalspecimens.html.

49. https://www.ifcc.org/ifcc-news/2020-03-26-ifcc-information-guideon-covid-19/.

50. fda.gov/media/136314/download.

51. Zhao J, Yuan $\mathrm{Q}$, Wang $\mathrm{H}$, et al. Antibody responses to SARS-CoV-2 in patients of novel coronavirus disease 2019. Clin Infect Dis 2020;ciaa344.

52. https://www.fda.gov/media/136522/download.

53. Lurie N, Saville M, Hatchett R, et al. Developing COVID-19 vaccines at pandemic speed. N Engl J Med 2020;382(21):1969-1973. DOI: 10.1056/ NEJMp2005630.

54. Kwok KO, Lai F, Wei WI, et al. Herd immunity - estimating the level required to halt the COVID-19 epidemics in affected countries. J Infect 2020;80(6):e32-e33. DOI: 10.1016/j.jinf.2020.03.027pii: S0163$4453(20) 30154-7$ 